VP-4

\title{
Laparoscopic Sg 2 partial resection due to recurrent colorectal metastasis after open right hepatectomy: Video case report
}

\author{
Denys FEDOROV*, Oleksandr OSTAPYSHEN, Olena PIDOPRYHORA
}

Liver Transplantation and Surgery Department, National Institute of Surgery and Transplantation, Kyiv, Ukraine

Introduction: Liver resection in combination with modern chemotherapy is effective way to treat colorectal liver metastases. Based on randomized trials results laparoscopic liver resection has benefits compared with open in terms of mortality and morbidity levels. Repeated liver resections are more complex procedures, but they also may be performed in laparoscopic way.

Methods: We present case of 48-year-old female patient with 6-year history of sigmoid cancer. The patient had left colectomy followed by 6 FOLFOX courses. Four years after she presented with liver metastases in right liver and open right hepatectomy was made. After 2 years of uneventful follow-up, she was referred to our hospital with signs of metastatic tumor located in Sg 2 of liver remnant. Percutaneous thermal ablation was thought to be contraindicated due to close relation of tumor to diaphragm and pericardium and after discussion on MDT partial laparoscopic liver resection of Sg 2 was performed.

Results: The procedure last for 186 minutes, mostly because of precise viscerolysis. Left hemyliver was carefully detached from dense adhesions with adjacent organs. The Pringle maneuver was not used and estimated blood loss was $120 \mathrm{~mL}$. Postoperative period was uneventful, patient was discharged on postoperative day 4. R0-resection margin was confirmed by pathologists. No tumor recurrence was observed on 1-year follow-up.

Conclusions: Laparoscopic approach is accessible option for parenchyma sparing resections of colorectal liver metastasis even after previous open live surgery. 